\title{
Patterns of sociodemographic and food practice characteristics in relation to fruit and vegetable consumption in children: results from the UK National Diet and Nutrition Survey Rolling Programme (2008-2010)
}

\author{
Tsz N Mak ${ }^{1, *}$, Celia J Prynne ${ }^{1}$, Darren Cole ${ }^{1}$, Emily Fitt ${ }^{1}$, Beverley Bates ${ }^{2}$ and \\ Alison M Stephen ${ }^{1}$ \\ ${ }^{1}$ MRC Human Nutrition Research, Elsie Widdowson Laboratory, 120 Fulbourn Road, Cambridge CB1 9NL, UK: \\ ${ }^{2}$ National Centre for Social Research, London, UK
}

Submitted 4 October 2012: Final revision received 6 March 2013: Accepted 12 June 2013: First published online 7 August 2013

\begin{abstract}
Objective: Few studies have considered the combined effects of home-related determinants on children's diet. The present study investigated independent associations between sociodemographic and food practice (SFP) characteristics and fruit and vegetable consumption in UK children and the combined effects of SFP on consumption using pattern analysis.

Design: Diet was assessed using $4 \mathrm{~d}$ food diaries, SFP were collected using computer-assisted personal interview. Linear regressions were used to test associations; principal component analysis was used to identify patterns of SFP characteristics. Regression of fruit $(\mathrm{g} / \mathrm{d})$ and vegetables $(\mathrm{g} / \mathrm{d}) v$. component scores of each pattern were performed.

Setting: UK National Diet and Nutrition Survey Rolling Programme (2008-2010). Subjects: Children aged 1·5-10 years ( $n$ 642).

Results: Significant associations were found between fruit and vegetable consumption and household socio-economic status. Pattern 1, which was positively correlated with household structure characteristics, was associated with increased fruit consumption $(P<0 \cdot 001)$. Pattern 2 , characterised by positive correlations for socio-economic status, fruit availability and organic food purchase, and negatively correlated with household size and the number of children per household, was associated with higher fruit and vegetable consumption (both $P<0 \cdot 001)$. Pattern 3, characterised by high frequency of eating out and eating takeaway, was associated with a lower consumption of both fruit $(P<0 \cdot 012)$ and vegetables $(P<0 \cdot 023)$.

Conclusions: Patterns of SFP determinants may be more informative than individual characteristics in relation to dietary outcomes. Results have public health implications on the healthfulness of meals eaten out of home and in takeaways, as well as the need to reduce diet inequality in larger households with lower socio-economic status.
\end{abstract}

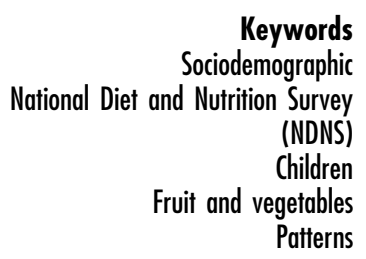

Determinants of eating behaviour and dietary intake in children have been extensively researched, with increasing understanding of influences such as the food environment, particularly at home, where children eat most often ${ }^{(1)}$. A wide range of home-related factors such as socio-economic status (SES), affordability, availability at home and accessibility ${ }^{(2-5)}$, to parental factors such as parent's education, nutritional knowledge and parental intake $^{(3,5-9)}$ and individual factors such as food preferences $^{(7,8)}$, have all been shown to relate to diet quality in children.
The most researched and consistent determinant of diet quality is the household or individual SES, which includes factors such as income, education and occupation ${ }^{(10-12)}$. Those in lower socio-economic groups tend to be less educated with lower nutritional knowledge and reduced affordability of healthier foods ${ }^{(11,13)}$. The downstream result is poorer food choices and poorer diet quality, often reflected by low fruit and vegetable (FV) consumption $^{(14,15)}$. Because SES is such a strong determinant of FV consumption and diet quality, it may be possible that the relationships between home-related determinants 
and $\mathrm{FV}$ consumption are confounded or mediated by SES. Furthermore, home-related factors are likely to be correlated with each other ${ }^{(16)}$. Traditional methods to study these relationships such as multiple regression analyses have led to inconclusive results, and previous studies suggested that holistic approaches to examine combinations of related factors may be more effective in understanding dietary behaviours than individual factors alone ${ }^{(17,18)}$. This could be due to multicollinearity between variables investigated and over-adjustment of correlated confounding factors in multivariate models, leading to reduced statistical power to detect effects between exposures and diet outcomes. One way to explore the combined effects of factors rather than independent associations is to identify patterns of characteristics using principal component analysis (PCA).

The aim of the present study was to use pattern analysis to investigate associations between combinations of sociodemographic and food practice (SFP) characteristics in relation to $\mathrm{FV}$ consumption, in a nationally representative sample of UK children aged 1.5 to 10 years. SFP characteristics of children and their households were collected in the UK National Diet and Nutrition Survey (NDNS) 2008-2010, which included the following: household size, the number of adults and the number of children in the household; demographic characteristics including household SES, ethnicity and region; the age and sex of the main food provider of the participating children; as well as household food practice characteristics, including if organic foods were ever purchased, purchase frequencies of fruit and vegetables, fruit availability at home, eating out and eating takeaway. We hypothesised that the National Statistic Socio-economic Classification 5 (NSSEC5), an indicator of household SES based on the head of household's occupation and used in UK national surveys, was associated with these SFP factors. We also predicted that independent associations between SFP and FV consumption would attenuate when adjusted for NSSEC5 in the models. Finally, we explored empirically derived patterns of SFP using PCA, a data-driven technique to reduce the SFP variables to a smaller number of mutually exclusive patterns. These patterns were then further examined in relation to FV consumption.

\section{Methods}

\section{Study sample and design}

Data were collected from 642 children aged 1.5 to 10 years in the NDNS Rolling Programme between February 2008 and April 2010 (Years 1 and 2). The aim of NDNS is to assess food consumption, nutrient intakes and nutritional status of individuals aged $1.5+$ years across the UK, with a core sample of 1000 people per year (500 adults $(\geq 19$ years) and 500 children ( 1.5 to 18 years)). Detailed descriptions of the sampling procedure and design have been reported ${ }^{(19)}$. Briefly, sampling was based on a random selection of postcode sectors throughout the UK; a number of addresses per sector were invited for participation by interviewers, following an initial information letter. Up to one adult and one child were selected from each participating household and a child 'boost' of addresses was included to compensate for households with no children ${ }^{(19)}$. Individual non-response weights were applied to reduce bias from differential response at the household and individual interview, and adjustment made for age, sex and regional profiles of participating individuals ${ }^{(2)}$. NDNS was conducted according to the guidelines laid down in the Declaration of Helsinki and all procedures involving human subjects were approved by the Oxfordshire A Research Ethics Committee. Written informed consent was obtained from parents of the children who participated in the survey ${ }^{(21)}$.

\section{Dietary assessment}

Unweighed food diaries were used to record all food and beverages consumed over four consecutive days, with instructions given by trained interviewers at the initial visit. For bought foods such as ready meals and snacks, participants were asked to record brand names to aid identification during the coding process; for homemade dishes, recipes were recorded separately with detailed description of ingredients, quantities and cooking methods. Portion sizes were estimated using household measures and weights from food package labels. Further description and justification of the dietary assessment have been reported elsewhere ${ }^{(21,22)}$. For this age group, parents and carers filled in the food diaries, with help from other people who had looked after the children ${ }^{(22)}$. The interviewer made a brief visit (either via telephone or in person) during the recording period to check for compliance and answer queries. Upon completion of the diary, the interviewer returned to collect the food diary and checked for any missing information ${ }^{(22)}$. To be considered fully productive, participants had to record their intakes for three days or more. Food diaries were returned to the Medical Research Council - Human Nutrition Research and were coded by trained diet coders using the in-house dietary assessment software, Diet in Nutrients out, with nutrient values provided by the UK NDNS Nutrient Databank.

\section{Disaggregation of fruit and vegetable consumption}

Portion sizes of discrete fruit items and discrete vegetable items were determined using the publication Food Portion Sizes $^{(23)}$. To quantify consumption of fruit and vegetables more accurately, disaggregation of mixed dishes was undertaken. The method adopted to disaggregate food codes in NDNS has been described previously ${ }^{(24)}$. Essentially, proportions of fruit and vegetables in composite dishes were calculated in three main ways: (i) manufactured product information from packaging; (ii) standard recipes from 
McCance and Widdowson's The Composition of Foods ${ }^{(25)}$; and (iii) homemade recipes from respondents' food diaries. For products that contain dried fruit and vegetables, such as soup powders, dried FV content was scaled up using the water content of the fresh equivalent. The amounts of disaggregated and discrete fruit and vegetables consumed by each participant per day were summed and the mean intakes of fruit $(\mathrm{g} / \mathrm{d})$ and vegetables $(\mathrm{g} / \mathrm{d})$ over the recording period were calculated per person.

\section{Computer-assisted personal interview}

A computer-assisted personal interview (CAPI) was conducted face-to-face by the interviewer during the initial visit to the participant's home. The CAPI consisted of three main elements: (i) a household structure interview; (ii) a main food provider (MFP) interview; and (iii) an individual interview. Together these gathered information on demographics, household composition, shopping and food preparation practices, cooking skills and facilities at home. Information on employment and income of the household reference person, defined as the person in whose name the property is owned or rented with the highest income, was used to determine the socio-economic classification of the household ${ }^{(21)}$. For this age group, the questions in the CAPI were answered on behalf of the child by the MFP, defined as the person best placed to answer questions about food purchase and preparation for the participant, who was usually the mother of the child. Details of the CAPI and the full questionnaire have been reported ${ }^{(19,26)}$.

\section{Sociodemographic variables}

Variables relating to sociodemographic characteristics and household structure were collected in the first part of the CAPI. NSSEC5 was used as the household SES indicator for the survey ${ }^{(27)}$, which was based on occupation and included five sub-categories: (i) managerial and professional; (ii) intermediates; (iii) small employers and own account workers; (iv) lower supervisory and technical; and (v) routine, manual, never worked, long-term unemployment. Since 2001, NSSEC has been used as the main measure of household SES for all official statistics and surveys in the UK. NSSEC takes into account details of employment status of the household reference person (whether an employer, self-employed or employee; whether a supervisor, manager, etc.), and is seen as superior to other social classification measures as it has been constructed to determine employment relations and conditions of occupation, to show the structure of socio-economic position in modern societies and to explain variations in social behaviour and other social phenomena ${ }^{(28)}$. Ethnicity was recorded as white or non-white. Six categories were used to describe region: (i) England: North; (ii) England: Central/Midlands; (iii) England: South (including London); (iv) Scotland; (v) Wales; and (vi) Northern Ireland. For household structure, information was collected on: the size of the household (on the scale of 2, 3, 4 and 5+); the number of adults per household (1, 2 and 3+); and the number of children per household (1, 2, 3 and 4+). The level of household income was asked in the questionnaire, but due to a high percentage of non-responses, this variable was not used in the analysis. Information on parent's education level was not collected in NDNS. For adults participating in NDNS, questions related to education were asked but not for children, and therefore this variable could not be used in the current analysis.

\section{Food practice variables}

The MFP interview in the CAPI captured a number of variables related to food practices at home. Specific fruit and vegetable indicators were recorded, including frequency of fruit and vegetable purchase (' $<3$ times per month', 'weekly', '2 or 3 times per week', 'daily') and frequency of fruit availability at home ('never', 'weekly', 'most of the time'). Respondents were also asked if they have ever purchased organic foods (yes, no), how frequently they eat out ('rarely or never', 'once or twice per month', 'more than once per week') and the frequency of eating takeaway ('rarely or never', 'once or twice per month', 'more than once per week'). Age and sex of the MFP were included in the analyses.

\section{Statistical analysis}

All analyses were performed using the Stata statistical software package release 11. Descriptive statistics of continuous variables were expressed as means and standard deviations. Distributions of the categorical variables were described in absolute frequencies and percentages. The $\chi^{2}$ test was used to test for associations between NSSEC5 and individual SFP variables, except for MFP age, which was a continuous variable; ANOVA was used instead to test for association. Independent associations between the SFP variables and fruit consumption $(\mathrm{g} / \mathrm{d})$ and vegetable $(\mathrm{g} / \mathrm{d})$ consumption were determined using linear regression analysis, with all models adjusted for age and sex of the child. Patterns of the variables were identified using PCA. PCA is a statistical data-reduction technique that reduces a large number of variables into a smaller number of uncorrelated linear combinations of variables that contain most of the variance of the sample ${ }^{(29)}$. PCA is scale sensitive $^{(30)}$ and only variables of continuous, ordinal and binary nature were included in the analysis; hence the variable 'region' was removed from analyses. The variable MFP sex was also removed since only $7 \%$ of MFP were male. Eleven variables were therefore entered in the PCA: household size, number of adults per household, number of children per household, NSSEC5, ethnicity, MFP age, frequency of fruit availability, frequency of FV purchase, organic purchase, frequency of eating out and frequency of eating takeaway. Variables with component loadings $> \pm 0 \cdot 3$ were considered to be correlated highly with the construction of the pattern. The screeplot was 
used to decide on the number of patterns to retain for subsequent analyses. Pattern scores were calculated for each participant for each pattern retained and were regressed $v$. fruit $(\mathrm{g} / \mathrm{d})$ and vegetable $(\mathrm{g} / \mathrm{d})$ consumption. Age and sex of the child were adjusted in the final models. Survey commands (svy) were used throughout to account for the multistage survey design and non-response weighting was applied in the regression analyses.

\section{Results}

\section{Sample characteristics}

Table 1 shows the basic characteristics of the sample population. Fifty-one per cent of children were male. Mean household size was $3 \cdot 8(\mathrm{SD} 1 \cdot 1)$ persons, with two adults (SD $0 \cdot 7)$ and two children (SD $0 \cdot 8$ ) on average. The majority of the sample was white, from England South ( $40.5 \%$ ), and $41 \%$ of the household reference persons had managerial and professional occupations. In terms of food practice characteristics, the majority of the MFP were female, with a mean age of $35(\mathrm{SD} 7 \cdot 2)$ years. Most MFP purchased FV weekly or 2 or 3 times per week, and indicated that fruit were available at home most of the time. About $70 \%$ of children ate out at least once per month, while approximately $50 \%$ of children rarely or never ate takeaway.

\section{Housebold socio-economic status and sociodemographic and food practice characteristics}

NSSEC5 was associated with seven out of twelve SFP variables (Table 2), including the number of adults per household, MFP age, frequency of fruit availability, organic food purchase (all $P<0 \cdot 001$ ), household size, frequency of eating takeaway (both $P<0 \cdot 01$ ) and frequency of FV purchase $(P<0 \cdot 05)$.

\section{Independent associations between sociodemographic and food practice characteristics and fruit and vegetable consumption}

Child's age was associated with fruit consumption and vegetable consumption, where fruit consumption decreased as age increased $(P=0 \cdot 002)$ with the reverse seen for vegetables $(P<0 \cdot 001$; Table 3$)$. Compared with singleadult households (reference), children in households with two adults consumed approximately $21 \cdot 3 \mathrm{~g}$ more fruit per day $(P=0 \cdot 01)$; however the relationship was not significant when adjusted for NSSEC5. On the other hand, vegetable consumption was $29.7 \mathrm{~g} / \mathrm{d}$ lower $(P=0.037)$ in households with more than three adults after adjusting for NSSEC5. Differences in consumption were seen in different household socio-economic classifications, where children in the highest NSSEC5 group (managerial and professional) consumed significantly more fruit and vegetables daily (both $P<0 \cdot 001$ ) than those from the lowest NSSEC5 group (reference). Other sociodemographic characteristics were not significantly related to FV consumption.

Associations were seen between food practice characteristics and FV consumption in children (Table 4) and most remained significant after adjusting for NSSEC5. Fruit consumption increased as MFP age increased $(P=0 \cdot 007)$. Those who reported to have ever bought organic food consumed $24.8 \mathrm{~g}$ more fruit $(P=0.001)$ and $14.6 \mathrm{~g}$ more vegetables $(P<0 \cdot 019)$ per day than those who had never purchased organic food (reference) after adjusting for NSSEC5 and age and sex of children (fully adjusted model). Higher fruit consumption was also seen in children in the fully adjusted model where the MFP reported to purchase fruit and vegetables frequently (2 or 3 times per week $(P=0 \cdot 001)$ or once daily $(P=0 \cdot 025))$ and had fruit available at home weekly or most of the time (both $P<0 \cdot 001$ ). Those who ate takeaway more than once per week consumed $32 \cdot 2 \mathrm{~g}$ less fruit $(P<0 \cdot 001)$ and $20 \cdot 4 \mathrm{~g}$ less vegetables $(P=0 \cdot 028)$ per day than those rarely or never ate takeaway (reference) in the fully adjusted model.

\section{Pattern analysis}

Of the eleven principal components (patterns) identified from the PCA, three were retained for subsequent analyses, where they explained approximately $46 \%$ of total variance of the data. The loadings of each variable for the three patterns were shown in Table 5 .

The first pattern described greater correlations with household structure, where higher positive correlations were seen for household size, number of children and number of adults per household. For each unit of increase in pattern score, children significantly increased consumption of fruit by $9.5 \mathrm{~g} / \mathrm{d}(P<0 \cdot 001)$; no difference was observed for vegetables (Table 6 ). The second pattern was characterised by positive correlations for NSSEC5, fruit availability and organic food purchase, and in contrast to pattern 1 , this pattern was negatively correlated with household size and the number of children per household. For this pattern, fruit and vegetable consumption increased significantly by $16 \cdot 8 \mathrm{~g} / \mathrm{d}$ and $8 \cdot 1 \mathrm{~g} / \mathrm{d}$, respectively, per unit increase of pattern score (both $P<0 \cdot 001$ ). Lastly, the third pattern explained positive correlations with frequencies of eating out and eating takeaway, as well as negative correlation with ethnicity, indicating those who correlated higher with this pattern were more likely to be white than non-white. This pattern was associated with a lower consumption of both fruit $(P=0 \cdot 012)$ and vegetables $(P=0 \cdot 023)$.

\section{Discussion}

The present study has demonstrated household SES was significantly associated with a number of SFP variables in this nationally representative sample of UK children aged 1.5 to 10 years. There were very few associations 
Table 1 Characteristics of the sample population: children ( $n$ 642) aged 1.5-10 years, UK National Diet and Nutrition Survey Rolling Programme (2008-2010)

\begin{tabular}{|c|c|c|c|}
\hline & $n$ & & $\%$ \\
\hline \multicolumn{4}{|l|}{ Sociodemographic characteristics } \\
\hline \multicolumn{4}{|l|}{ Child's age group (years) } \\
\hline $1 \cdot 5-3$ & 219 & & $34 \cdot 1$ \\
\hline $4-6$ & 192 & & $29 \cdot 9$ \\
\hline $7-10$ & 231 & & $36 \cdot 0$ \\
\hline \multicolumn{4}{|l|}{ Child's sex } \\
\hline Male & 327 & & $50 \cdot 9$ \\
\hline \multicolumn{4}{|l|}{ Household size } \\
\hline Mean & & $3 \cdot 8$ & \\
\hline SD & & $1 \cdot 1$ & \\
\hline 2 & 43 & & $6 \cdot 7$ \\
\hline 3 & 213 & & $33 \cdot 2$ \\
\hline 4 & 256 & & 39.9 \\
\hline $5+$ & 130 & & $20 \cdot 3$ \\
\hline \multicolumn{4}{|l|}{ Number of adults in household } \\
\hline Mean & & $2 \cdot 0$ & \\
\hline SD & & $0 \cdot 7$ & \\
\hline 1 & 106 & & $16 \cdot 5$ \\
\hline 2 & 479 & & $74 \cdot 6$ \\
\hline $3+$ & 57 & & 8.9 \\
\hline \multicolumn{4}{|l|}{ Number of children in household } \\
\hline Mean & & 1.9 & \\
\hline SD & & $0 \cdot 8$ & \\
\hline 1 & 228 & & $35 \cdot 5$ \\
\hline 2 & 300 & & $46 \cdot 7$ \\
\hline 3 & 93 & & $14 \cdot 5$ \\
\hline $4+$ & 21 & & $3 \cdot 3$ \\
\hline \multicolumn{4}{|l|}{ NSSEC5 } \\
\hline Managerial and professional & 263 & & $41 \cdot 0$ \\
\hline Intermediates & 50 & & $7 \cdot 8$ \\
\hline Small employers and own account workers & 77 & & $12 \cdot 0$ \\
\hline Lower supervisory and technical & 68 & & $10 \cdot 6$ \\
\hline Routine, manual, never worked, long-term unemployment & 184 & & $28 \cdot 7$ \\
\hline \multicolumn{4}{|l|}{ Ethnicity } \\
\hline White & 537 & & $83 \cdot 6$ \\
\hline Non-white & 105 & & $16 \cdot 4$ \\
\hline \multicolumn{4}{|l|}{ Region } \\
\hline England: North & 158 & & $24 \cdot 6$ \\
\hline England: Central/Midlands & 107 & & $16 \cdot 7$ \\
\hline England: South (incl. London) & 260 & & $40 \cdot 5$ \\
\hline Scotland & 49 & & $7 \cdot 6$ \\
\hline Wales & 46 & & $7 \cdot 2$ \\
\hline Northern Ireland & 22 & & $3 \cdot 4$ \\
\hline \multicolumn{4}{|l|}{ Home characteristics } \\
\hline \multicolumn{4}{|l|}{ MFP sex } \\
\hline Female & 597 & & $93 \cdot 0$ \\
\hline \multicolumn{4}{|l|}{ MFP age (years) } \\
\hline Mean & & $34 \cdot 9$ & \\
\hline SD & & $7 \cdot 1$ & \\
\hline Frequency of purchasing FV & & & \\
\hline$<3$ times/month & 29 & & 4.5 \\
\hline Weekly & 295 & & $46 \cdot 0$ \\
\hline 2 or 3 times per week & 307 & & $47 \cdot 8$ \\
\hline Daily & 11 & & $1 \cdot 7$ \\
\hline Frequency of fruit availability at home & & & \\
\hline Never & 2 & & $0 \cdot 3$ \\
\hline Weekly & 46 & & $7 \cdot 2$ \\
\hline Most of the time & 594 & & 92.5 \\
\hline Purchase organic food & & & \\
\hline No & 333 & & 51.9 \\
\hline Yes & 309 & & $48 \cdot 1$ \\
\hline Frequency of eating out & & & \\
\hline Rarely or never & 198 & & $30 \cdot 8$ \\
\hline Once or twice per month & 319 & & $49 \cdot 7$ \\
\hline More than once per week & 125 & & $19 \cdot 5$ \\
\hline Frequency of eating takeaway & & & \\
\hline Rarely or never & 322 & & $50 \cdot 2$ \\
\hline Once or twice per month & 217 & & $33 \cdot 8$ \\
\hline More than once per week & 103 & & $16 \cdot 0$ \\
\hline
\end{tabular}

NSSEC5, National Statistic Socio-economic Classification 5; MFP, main food provider; FV, fruit and vegetables. 
Table 2 Associations between NSSEC5 and SFP characteristics: children ( $n$ 642) aged 1.5-10 years, UK National Diet and Nutrition Survey Rolling Programme (2008-2010)

\begin{tabular}{|c|c|c|c|c|c|c|c|}
\hline \multirow[b]{3}{*}{ Sociodemographic characteristics } & \multicolumn{6}{|c|}{ NSSEC5* (lowest to highest) } & \multirow[b]{3}{*}{$P$ valuet } \\
\hline & 1 & 2 & 3 & 4 & 5 & Total & \\
\hline & \multicolumn{6}{|c|}{$\%$} & \\
\hline \multicolumn{8}{|l|}{ Child's age group (years) } \\
\hline $1.5-3(n 219)$ & $28 \cdot 8$ & $9 \cdot 6$ & $11 \cdot 9$ & $8 \cdot 2$ & $41 \cdot 6$ & $100 \cdot 0$ & \\
\hline $4-6(n 192)$ & $27 \cdot 1$ & $11 \cdot 5$ & $10 \cdot 9$ & $6 \cdot \overline{8}$ & $43 \cdot 8$ & $100 \cdot 0$ & \\
\hline $7-10(n 231)$ & $29 \cdot 9$ & $10 \cdot 8$ & $13 \cdot 0$ & $8 \cdot 2$ & $38 \cdot 1$ & $100 \cdot 0$ & \\
\hline Total (n 642) & $28 \cdot 7$ & $10 \cdot 6$ & $12 \cdot 0$ & $7 \cdot 8$ & $41 \cdot 0$ & $100 \cdot 0$ & 0.973 \\
\hline \multicolumn{8}{|l|}{ Child's sex } \\
\hline Male ( $n$ 327) & $25 \cdot 7$ & $12 \cdot 2$ & $11 \cdot 3$ & $7 \cdot 3$ & $43 \cdot 4$ & $100 \cdot 0$ & \\
\hline Female ( $n$ 315) & $31 \cdot 8$ & 8.9 & $12 \cdot 7$ & $8 \cdot 3$ & $38 \cdot 4$ & $100 \cdot 0$ & \\
\hline Total (n 642) & $28 \cdot 7$ & $10 \cdot 6$ & $12 \cdot 0$ & $7 \cdot 8$ & $41 \cdot 0$ & $100 \cdot 0$ & $0 \cdot 271$ \\
\hline \multicolumn{8}{|l|}{ Household size } \\
\hline $2(n 43)$ & $39 \cdot 5$ & $18 \cdot 6$ & $4 \cdot 7$ & $18 \cdot 6$ & $18 \cdot 6$ & $100 \cdot 0$ & \\
\hline $3(n 213)$ & $28 \cdot 6$ & $9 \cdot 4$ & $9 \cdot 9$ & 8.9 & $43 \cdot 2$ & $100 \cdot 0$ & \\
\hline $4(n 256)$ & $27 \cdot 7$ & $11 \cdot 3$ & $11 \cdot 3$ & $6 \cdot 3$ & $43 \cdot 4$ & $100 \cdot 0$ & \\
\hline $5+(n 130)$ & $26 \cdot 9$ & $8 \cdot 5$ & $19 \cdot 2$ & $5 \cdot 4$ & $40 \cdot 0$ & $100 \cdot 0$ & \\
\hline Total $(n 642)$ & $28 \cdot 7$ & $10 \cdot 6$ & $12 \cdot 0$ & $7 \cdot 8$ & $41 \cdot 0$ & $100 \cdot 0$ & 0.005 \\
\hline \multicolumn{8}{|l|}{ Number of adults in household } \\
\hline $1(n 106)$ & $51 \cdot 9$ & $12 \cdot 3$ & $3 \cdot 8$ & $15 \cdot 1$ & $17 \cdot 0$ & $100 \cdot 0$ & \\
\hline $2(n 479)$ & $23 \cdot 2$ & $10 \cdot 4$ & $13 \cdot 2$ & $6 \cdot 1$ & $47 \cdot 2$ & $100 \cdot 0$ & \\
\hline $3+(n 57)$ & $31 \cdot 6$ & $8 \cdot 8$ & $17 \cdot 5$ & $8 \cdot 8$ & $33 \cdot 3$ & $100 \cdot 0$ & \\
\hline Total $(n 642)$ & $28 \cdot 7$ & $10 \cdot 6$ & $12 \cdot 0$ & $7 \cdot 8$ & $41 \cdot 0$ & $100 \cdot 0$ & $<0.001$ \\
\hline \multicolumn{8}{|l|}{ Number of children in household } \\
\hline $1(n 228)$ & $24 \cdot 6$ & $13 \cdot 6$ & $9 \cdot 7$ & $9 \cdot 2$ & $43 \cdot 0$ & $100 \cdot 0$ & \\
\hline $2(n 300)$ & $32 \cdot 0$ & $8 \cdot 7$ & $12 \cdot 0$ & $7 \cdot 7$ & $39 \cdot 7$ & $100 \cdot 0$ & \\
\hline $3(n 93)$ & $25 \cdot 8$ & $7 \cdot 5$ & $16 \cdot 1$ & $5 \cdot 4$ & $45 \cdot 2$ & $100 \cdot 0$ & \\
\hline $4+(n 21)$ & $38 \cdot 1$ & $19 \cdot 1$ & $19 \cdot 1$ & $4 \cdot 8$ & $19 \cdot 1$ & $100 \cdot 0$ & \\
\hline Total ( $n$ 642) & $28 \cdot 7$ & $10 \cdot 6$ & $12 \cdot 0$ & $7 \cdot 8$ & $41 \cdot 0$ & $100 \cdot 0$ & $0 \cdot 163$ \\
\hline \multicolumn{8}{|l|}{ Ethnicity } \\
\hline White ( $n$ 537) & $27 \cdot 6$ & $9 \cdot 7$ & $12 \cdot 7$ & $8 \cdot 2$ & $41 \cdot 9$ & $100 \cdot 0$ & \\
\hline Non-white (n 105) & $34 \cdot 3$ & $15 \cdot 2$ & $8 \cdot 6$ & $5 \cdot 7$ & $36 \cdot 2$ & $100 \cdot 0$ & \\
\hline Total $(n 642)$ & $28 \cdot 7$ & $10 \cdot \overline{6}$ & $12 \cdot 0$ & $7 \cdot 8$ & $41 \cdot 0$ & $100 \cdot 0$ & $0 \cdot 161$ \\
\hline \multicolumn{8}{|l|}{ Region } \\
\hline England: North ( $n$ 158) & $33 \cdot 5$ & $8 \cdot 9$ & $6 \cdot 3$ & $7 \cdot 6$ & $43 \cdot 7$ & $100 \cdot 0$ & \\
\hline England: Central/Midlands ( $n$ 107) & $28 \cdot 0$ & $11 \cdot 2$ & $15 \cdot 0$ & $10 \cdot 3$ & $35 \cdot 5$ & $100 \cdot 0$ & \\
\hline England: South (incl. London) ( $n$ 260) & $25 \cdot 0$ & $10 \cdot 0$ & $14 \cdot 2$ & $7 \cdot 3$ & $43 \cdot 5$ & $100 \cdot 0$ & \\
\hline Scotland $(n 49)$ & $28 \cdot 6$ & $12 \cdot 2$ & $8 \cdot 2$ & $6 \cdot 1$ & $44 \cdot 9$ & $100 \cdot 0$ & \\
\hline Wales (n 46) & $28 \cdot 3$ & $15 \cdot 2$ & $17 \cdot 4$ & $6 \cdot 5$ & $32 \cdot 6$ & $100 \cdot 0$ & \\
\hline Northern Ireland (n 22) & $40 \cdot 9$ & $13 \cdot \overline{6}$ & $9 \cdot 1$ & $9 \cdot 1$ & $27 \cdot 3$ & $100 \cdot 0$ & \\
\hline \multirow[t]{2}{*}{ Total $(n$ 642) } & $28 \cdot 7$ & $10 \cdot 6$ & $12 \cdot 0$ & $7 \cdot 8$ & $41 \cdot 0$ & $100 \cdot 0$ & 0.576 \\
\hline & \multicolumn{6}{|c|}{ NSSEC5* (lowest to highest) } & \\
\hline Food practice characteristics & 1 & 2 & 3 & 4 & 5 & Total & $P$ value \\
\hline \multicolumn{8}{|l|}{ MFP age (years) } \\
\hline Mean & $32 \cdot 4$ & $34 \cdot 1$ & $36 \cdot 1$ & $34 \cdot 0$ & $36 \cdot 6$ & $34 \cdot 9$ & $<0.001 \ddagger$ \\
\hline SD & $7 \cdot 8$ & $6 \cdot 6$ & $7 \cdot 5$ & $6 \cdot 6$ & $6 \cdot 0$ & $7 \cdot 1$ & \\
\hline$n$ & 184 & 68 & 77 & 50 & 263 & 642 & \\
\hline & & & & & & & \\
\hline MFP sex & & & & & & & \\
\hline Male ( $n$ 45) & $24 \cdot 4$ & $13 \cdot 3$ & $6 \cdot 7$ & $11 \cdot 1$ & $44 \cdot 4$ & $100 \cdot 0$ & \\
\hline Female ( $n$ 597) & $29 \cdot 0$ & $10 \cdot 4$ & $12 \cdot 4$ & $7 \cdot 5$ & $40 \cdot 7$ & $100 \cdot 0$ & \\
\hline Total $(n 642)$ & $28 \cdot 7$ & $10 \cdot 6$ & $12 \cdot 0$ & $7 \cdot 8$ & $41 \cdot 0$ & $100 \cdot 0$ & 0.624 \\
\hline Frequency of purchasing FV & & & & & & & \\
\hline$<3$ times per month ( $n$ 29) & $37 \cdot 9$ & $6 \cdot 9$ & $20 \cdot 7$ & $10 \cdot 3$ & $24 \cdot 1$ & $100 \cdot 0$ & \\
\hline Weekly (n 295) & $30 \cdot 9$ & $14 \cdot 2$ & $9 \cdot 8$ & $6 \cdot 8$ & $38 \cdot 3$ & $100 \cdot 0$ & \\
\hline 2 or 3 times per week $(n 307)$ & $26 \cdot 1$ & $7 \cdot \overline{8}$ & $13 \cdot 7$ & $8 \cdot 8$ & $43 \cdot 7$ & $100 \cdot 0$ & \\
\hline Daily $(n 11)$ & $18 \cdot 2$ & 0.0 & 0.0 & 0.0 & $81 \cdot 8$ & $100 \cdot 0$ & \\
\hline Total $(n 642)$ & $28 \cdot 7$ & $10 \cdot 6$ & $12 \cdot 0$ & $7 \cdot 8$ & $41 \cdot 0$ & $100 \cdot 0$ & 0.018 \\
\hline Frequency of fruit availability at home & & & & & & & \\
\hline Never $(n 2)$ & $50 \cdot 0$ & $0 \cdot 0$ & $0 \cdot 0$ & $50 \cdot 0$ & $0 \cdot 0$ & $100 \cdot 0$ & \\
\hline Weekly ( $n$ 46) & $60 \cdot 9$ & $10 \cdot 9$ & $8 \cdot 7$ & $10 \cdot 9$ & $8 \cdot 7$ & $100 \cdot 0$ & \\
\hline Most of the time ( $n$ 594) & $26 \cdot 1$ & $10 \cdot 6$ & $12 \cdot 3$ & $7 \cdot 4$ & $43 \cdot 6$ & $100 \cdot 0$ & \\
\hline Total $(n 642)$ & $28 \cdot 7$ & $10 \cdot 6$ & $12 \cdot 0$ & $7 \cdot 8$ & $41 \cdot 0$ & $100 \cdot 0$ & $<0.001$ \\
\hline
\end{tabular}




\begin{tabular}{|c|c|c|c|c|c|c|c|}
\hline \multirow[b]{3}{*}{ Food practice characteristics } & \multicolumn{6}{|c|}{ NSSEC5* (lowest to highest) } & \multirow[b]{3}{*}{$P$ value } \\
\hline & 1 & 2 & 3 & 4 & 5 & Total & \\
\hline & \multicolumn{6}{|c|}{$\%$} & \\
\hline \multicolumn{8}{|l|}{ Purchase organic food } \\
\hline No $(n 333)$ & $39 \cdot 6$ & $10 \cdot 8$ & $10 \cdot 2$ & $9 \cdot 9$ & $29 \cdot 4$ & $100 \cdot 0$ & \\
\hline Yes $(n 309)$ & $16 \cdot 8$ & $10 \cdot 4$ & $13 \cdot 9$ & $5 \cdot 5$ & $53 \cdot 4$ & $100 \cdot 0$ & \\
\hline Total ( $n$ 642) & $28 \cdot 7$ & $10 \cdot 6$ & $12 \cdot 0$ & $7 \cdot 8$ & $41 \cdot 0$ & $100 \cdot 0$ & $<0.001$ \\
\hline \multicolumn{8}{|l|}{ Frequency of eating out } \\
\hline Rarely or never ( $n$ 198) & $35 \cdot 4$ & $11 \cdot 6$ & $12 \cdot 6$ & $8 \cdot 6$ & $31 \cdot 8$ & $100 \cdot 0$ & \\
\hline Once or twice per month ( $n$ 319) & $25 \cdot 4$ & $11 \cdot 0$ & $11 \cdot 3$ & $8 \cdot 5$ & $43 \cdot 9$ & $100 \cdot 0$ & \\
\hline More than once per week $(n 125)$ & $26 \cdot 4$ & $8 \cdot 0$ & $12 \cdot 8$ & $4 \cdot 8$ & $48 \cdot 0$ & $100 \cdot 0$ & \\
\hline Total $(n$ 642) & $28 \cdot 7$ & $10 \cdot 6$ & $12 \cdot 0$ & $7 \cdot 8$ & $41 \cdot 0$ & $100 \cdot 0$ & 0.087 \\
\hline \multicolumn{8}{|l|}{ Frequency of eating takeaway } \\
\hline Rarely or never ( $n$ 322) & $22 \cdot 1$ & $9 \cdot 9$ & $11 \cdot 8$ & $7 \cdot 8$ & $48 \cdot 5$ & $100 \cdot 0$ & \\
\hline Once or twice per month $(n 217)$ & $32 \cdot 7$ & $10 \cdot 1$ & $12 \cdot 0$ & $8 \cdot 8$ & $36 \cdot 4$ & $100 \cdot 0$ & \\
\hline More than once per week $(n$ 103) & $40 \cdot 8$ & $13 \cdot 6$ & $12 \cdot 6$ & $5 \cdot 8$ & $27 \cdot 2$ & $100 \cdot 0$ & \\
\hline Total $(n$ 642) & $28 \cdot 7$ & $10 \cdot 6$ & $12 \cdot 0$ & $7 \cdot 8$ & $41 \cdot 0$ & $100 \cdot 0$ & 0.003 \\
\hline
\end{tabular}

NSSEC5, National Statistic Socio-economic Classification 5; SFP, sociodemographic and food practice; MFP, main food provider; FV, fruit and vegetables. ${ }^{*}$ NSSEC5 categories were as follows: 1 = routine, manual, never worked, long-term unemployment; $2=$ lower supervisory and technical occupations; $3=$ small employers and own account workers; 4 = intermediate occupations; $5=$ managerial and professional occupations.

tAssociations between SFP characteristics and NSSEC5 examined using $\chi^{2}$ tests. $P$ values significant at $P<0 \cdot 05$.

$\ddagger$ Association between MFP age and NSSEC5 tested using ANOVA. $P$ value significant at $P<0.05$.

between sociodemographic variables and FV consumption, but several food practice characteristics were significantly related to $\mathrm{FV}$ consumption independent of household SES. Finally, through PCA, three distinct patterns of SFP characteristics were identified, where each pattern was uniquely associated with FV consumption.

While our results have shown that household SES is indeed a strong predictor of $\mathrm{FV}$ consumption, especially when comparing the highest NSSEC5 group with the lowest group, our hypothesis that associations between SFP characteristics and FV consumption were confounded by SES was only partially supported. Attenuation of the significance level was seen for fruit consumption in relation to only two variables after adjustment for NSSEC5 ('two adults per household' and 'takeaway once or twice per month'), while vegetable consumption became significantly associated with 'three or more adults per household'. The majority of the food practice characteristics remained significantly associated with FV consumption even after adjusting for NSSEC5. Therefore, several food practice characteristics were important in predicting $\mathrm{FV}$ consumption irrespective of household socio-economic situation. Factors other than household SES may be important in driving food practices and decisions to provide fruit and vegetables to children. As suggested by previous studies, these could be parent's education, nutritional knowledge and income or affordability, but they were not tested in the current analysis.

Our results suggest that children from households where fruit and vegetables were frequently purchased have fruit frequently available at home, infrequently eat takeaway, and have higher daily FV consumption. Similar findings are seen in the literature on home food environment, where home availability and accessibility of fruit and vegetables are important determinants of consumption ${ }^{(5,31)}$, while high availability of unhealthy foods is associated with higher consumption of those ${ }^{(8,32)}$. It has been suggested that adolescents in two-parent families have better eating behaviours such as consumption of fruit and vegetables and regular breakfast compared with single-parent families $^{(4,33)}$, although studies did not control for the effects of SES on family structure. Our results also showed that two-adult households had higher fruit consumption, although the relationship was no longer significant after adjusting for SES. It has been found previously that as age increases, children tend to consume less fruit as well as vegetables $^{(34)}$. Our findings suggested that children's age was inversely associated with fruit consumption only, but not for vegetables where a positive relationship was seen. The contrasting result may reflect different methodologies used in measuring FV consumption between studies. Some studies have measured fruit and vegetables together as one food category using FFQ or other means of dietary assessment, while in the present study they were assessed using food diaries, disaggregated from mixed dishes and analysed separately, as previous results indicate that fruit consumption and vegetable consumption are two distinct eating behaviours ${ }^{(35,36)}$.

Households with three adults or more appeared to be unfavourable for children's vegetable consumption independent of SES. To our knowledge, this has not been tested before. MFP age was found to be a strong positive predictor of FV consumption, which partially supports the finding from a recent study where maternal age was associated with higher pattern scores on the 'healthy' diet $^{(37)}$; however, at odds with another study on maternal age at delivery and food consumption in pre-school 
Table 3 Independent associations between sociodemographic characteristics and FV consumption: children ( $n$ 642) aged 1.5-10 years, UK National Diet and Nutrition Survey Rolling Programme (2008-2010)

\begin{tabular}{|c|c|c|c|c|c|c|c|c|c|c|c|c|}
\hline \multirow[b]{2}{*}{ Sociodemographic characteristics } & \multicolumn{3}{|c|}{ Fruit $^{*}$} & \multicolumn{3}{|c|}{ Fruitt } & \multicolumn{3}{|c|}{ Vegetables $^{*}$} & \multicolumn{3}{|c|}{ Vegetablest } \\
\hline & $\beta$ & $P$ value & $95 \% \mathrm{Cl}$ & $\beta$ & $P$ value & $95 \% \mathrm{Cl}$ & $\beta$ & $P$ value & $95 \% \mathrm{Cl}$ & $\beta$ & $P$ value & $95 \% \mathrm{Cl}$ \\
\hline Child's age & $-3 \cdot 55$ & 0.002 & $-5 \cdot 76,-1 \cdot 35$ & - & - & - & $5 \cdot 86$ & $<0.001$ & $3 \cdot 67,8.00$ & - & - & - \\
\hline \multicolumn{13}{|l|}{ Child's sex } \\
\hline Male & Reference & - & - & - & - & - & Reference & - & - & - & - & - \\
\hline Female & $6 \cdot 27$ & 0.417 & $-8 \cdot 95,21 \cdot 48$ & - & - & - & -0.68 & 0.917 & $-13 \cdot 50,12 \cdot 14$ & - & - & - \\
\hline \multicolumn{13}{|l|}{ Household size } \\
\hline 2 & Reference & - & - & Reference & - & - & Reference & - & - & Reference & - & - \\
\hline 3 & 11.59 & 0.441 & $-18 \cdot 04,41 \cdot 22$ & $6 \cdot 15$ & 0.676 & $-22 \cdot 86,35 \cdot 15$ & -3.23 & 0.755 & $-23 \cdot 67,17 \cdot 21$ & $-6 \cdot 84$ & 0.518 & $-27 \cdot 65,13 \cdot 98$ \\
\hline 4 & $18 \cdot 17$ & 0.232 & $-11 \cdot 71,48 \cdot 06$ & $11 \cdot 72$ & 0.423 & $-17 \cdot 10,40 \cdot 53$ & $-3 \cdot 54$ & 0.725 & $-23 \cdot 34,16 \cdot 27$ & $-7 \cdot 81$ & 0.454 & $-28 \cdot 36,12 \cdot 74$ \\
\hline $5+$ & $12 \cdot 68$ & 0.402 & $-17 \cdot 13,42 \cdot 49$ & $6 \cdot 45$ & 0.658 & $-22 \cdot 30,35 \cdot 20$ & $-9 \cdot 00$ & 0.387 & $-29 \cdot 49,11 \cdot 48$ & $-13 \cdot 13$ & 0.215 & $-33 \cdot 95,7 \cdot 70$ \\
\hline \multicolumn{13}{|l|}{ Number of adults in household } \\
\hline 1 & Reference & - & - & Reference & - & - & Reference & - & - & Reference & - & - \\
\hline 2 & $21 \cdot 27$ & 0.010 & $5 \cdot 11,37 \cdot 44$ & $10 \cdot 13 \ddagger$ & 0.249 & $-7 \cdot 15,27 \cdot 41$ & $-4 \cdot 82$ & 0.550 & $-20 \cdot 70,11 \cdot 06$ & $-14 \cdot 12$ & 0.089 & $-30 \cdot 41,2 \cdot 18$ \\
\hline $3+$ & $7 \cdot 95$ & 0.567 & $-19 \cdot 45,35 \cdot 34$ & $-2 \cdot 85$ & 0.836 & $-30 \cdot 10,24 \cdot 39$ & $-21 \cdot 99$ & $0 \cdot 134$ & $-50 \cdot 83,6 \cdot 85$ & $-29 \cdot 72 \S$ & 0.037 & $-57 \cdot 65,-1 \cdot 80$ \\
\hline \multicolumn{13}{|l|}{ Number of children in household } \\
\hline 1 & Reference & - & - & Reference & - & - & Reference & - & - & Reference & - & - \\
\hline 2 & $6 \cdot 13$ & 0.445 & $-9 \cdot 71,21 \cdot 98$ & $8 \cdot 20$ & 0.308 & $-7 \cdot 64,24 \cdot 04$ & 0.95 & 0.879 & $-11 \cdot 41,13 \cdot 31$ & 2.04 & 0.753 & $-10 \cdot 74,14 \cdot 82$ \\
\hline 3 & 3.61 & 0.742 & $-17 \cdot 99,25 \cdot 20$ & $2 \cdot 58$ & 0.812 & $-18 \cdot 79,23.96$ & -5.99 & 0.491 & $-23 \cdot 11,11 \cdot 13$ & $-6 \cdot 83$ & 0.422 & $-23.59,9.93$ \\
\hline $4+$ & $-7 \cdot 85$ & 0.567 & $-34 \cdot 88,19 \cdot 17$ & $-3 \cdot 61$ & 0.804 & $-32 \cdot 41,25 \cdot 18$ & $-8 \cdot 54$ & 0.588 & $-39 \cdot 65,22 \cdot 57$ & $-4 \cdot 83$ & 0.765 & $-36 \cdot 68,27 \cdot 02$ \\
\hline \multicolumn{13}{|l|}{ NSSEC5 } \\
\hline $\begin{array}{l}\text { Routine, manual, never worked, } \\
\text { long-term unemployment }\end{array}$ & Reference & - & - & - & - & - & Reference & - & - & - & - & - \\
\hline Lower supervisory and technical & $20 \cdot 40$ & 0.046 & $0 \cdot 38,40 \cdot 42$ & - & - & - & 9.97 & 0.350 & $-11 \cdot 03,30 \cdot 97$ & - & - & - \\
\hline $\begin{array}{l}\text { Small employers and own account } \\
\text { workers }\end{array}$ & $40 \cdot 04$ & 0.001 & $17 \cdot 40,62 \cdot 69$ & - & - & - & $15 \cdot 27$ & $0 \cdot 157$ & $-5 \cdot 94,36 \cdot 49$ & - & - & - \\
\hline Intermediates & $34 \cdot 23$ & 0.033 & $2 \cdot 77,65 \cdot 69$ & - & - & - & $4 \cdot 37$ & 0.645 & $-14 \cdot 32,23.06$ & - & - & - \\
\hline Managerial and professional & $39 \cdot 40$ & $<0.001$ & $24 \cdot 06,54 \cdot 75$ & - & - & - & $26 \cdot 11$ & $<0.001$ & $12 \cdot 07,40 \cdot 16$ & - & - & - \\
\hline \multicolumn{13}{|l|}{ Ethnicity } \\
\hline White & Reference & - & - & Reference & - & - & Reference & - & - & Reference & - & - \\
\hline Non-white & $-7 \cdot 96$ & 0.382 & $-25 \cdot 90,9 \cdot 97$ & $-6 \cdot 15$ & 0.478 & $-23 \cdot 24,10 \cdot 94$ & $2 \cdot 95$ & 0.734 & $-14 \cdot 18,20 \cdot 07$ & 4.67 & 0.585 & $-12 \cdot 21,21 \cdot 56$ \\
\hline \multicolumn{13}{|l|}{ Region } \\
\hline England: North & Reference & - & - & Reference & - & - & Reference & - & - & Reference & - & - \\
\hline England: Central/Midlands & $4 \cdot 22$ & 0.748 & $-21 \cdot 63,30 \cdot 06$ & $1 \cdot 42$ & 0.907 & $-22 \cdot 62,25 \cdot 46$ & 3.03 & 0.763 & $-16 \cdot 74,22 \cdot 80$ & 2.55 & 0.807 & $-18 \cdot 07,23 \cdot 17$ \\
\hline England: South (incl. London) & $7 \cdot 03$ & 0.485 & $-12 \cdot 80,26 \cdot 86$ & 3.43 & 0.712 & $-14 \cdot 90,21 \cdot 77$ & $-1 \cdot 02$ & 0.889 & $-15 \cdot 44,13 \cdot 40$ & $-2 \cdot 18$ & 0.766 & $-16 \cdot 60,12 \cdot 25$ \\
\hline Scotland & $18 \cdot 02$ & 0.215 & $-10 \cdot 56,46 \cdot 60$ & $17 \cdot 14$ & 0.268 & $-13 \cdot 33,47 \cdot 60$ & $-1 \cdot 21$ & 0.920 & $-24.95,22.53$ & -1.69 & 0.882 & $-24 \cdot 18,20 \cdot 81$ \\
\hline Wales & $4 \cdot 26$ & 0.759 & $-23 \cdot 15,31 \cdot 67$ & $1 \cdot 85$ & 0.880 & $-22 \cdot 37,26 \cdot 07$ & 15.06 & 0.353 & $-16.88,47 \cdot 01$ & $15 \cdot 66$ & 0.318 & $-15 \cdot 19,46 \cdot 51$ \\
\hline Northern Ireland & -9.97 & 0.586 & $-46 \cdot 08,26 \cdot 14$ & $-6 \cdot 91$ & 0.687 & $-40 \cdot 75,26 \cdot 93$ & $-15 \cdot 79$ & 0.501 & $-62 \cdot 03,30 \cdot 45$ & $-12 \cdot 89$ & 0.568 & $-57 \cdot 43,31 \cdot 65$ \\
\hline
\end{tabular}

FV, fruit and vegetable; NSSEC5, National Statistic Socio-economic Classification 5.

${ }^{*}$ Model adjusted for age and sex.

tModel adjusted for age, sex and NSSEC5.

$\ddagger$ Variable category became non-significant after adjusting for NSSEC5.

$\S$ Variable category became significant after adjusting for NSSEC5. 
Table 4 Independent associations between food practice characteristics and FV consumption: children ( $n$ 642) aged 1.5-10 years, UK National Diet and Nutrition Survey Rolling Programme (2008-2010)

\begin{tabular}{|c|c|c|c|c|c|c|c|c|c|c|c|c|}
\hline \multirow[b]{2}{*}{ Food practice characteristics } & \multicolumn{3}{|c|}{ Fruit $^{\star}$} & \multicolumn{3}{|c|}{ Fruitt } & \multicolumn{3}{|c|}{ Vegetables* } & \multicolumn{3}{|c|}{ Vegetablest } \\
\hline & $\beta$ & $P$ value & $95 \% \mathrm{Cl}$ & $\beta$ & $P$ value & $95 \% \mathrm{Cl}$ & $\beta$ & $P$ value & $95 \% \mathrm{Cl}$ & $\beta$ & $P$ value & $95 \% \mathrm{Cl}$ \\
\hline MFP age & $2 \cdot 23$ & $<0.001$ & $1 \cdot 11,3 \cdot 34$ & $1 \cdot 53$ & 0.007 & $0 \cdot 42,2 \cdot 64$ & $0 \cdot 30$ & 0.509 & $-0 \cdot 60,1 \cdot 20$ & -0.25 & 0.583 & $-1 \cdot 16,0 \cdot 66$ \\
\hline \multicolumn{13}{|l|}{ MFP sex } \\
\hline Male & Reference & - & - & Reference & - & - & Reference & - & - & Reference & - & - \\
\hline Female & $8 \cdot 64$ & $0 \cdot 378$ & $-10 \cdot 67,27 \cdot 96$ & 8.69 & 0.375 & $-10 \cdot 59,27 \cdot 97$ & $15 \cdot 37$ & 0.064 & $-0 \cdot 89,31 \cdot 63$ & $15 \cdot 83$ & 0.060 & $-0 \cdot 68,32 \cdot 33$ \\
\hline \multicolumn{13}{|l|}{ Frequency of purchasing FV } \\
\hline$<3$ times per month & Reference & - & - & Reference & - & - & Reference & - & - & Reference & - & ${ }^{-}$ \\
\hline Weekly & $21 \cdot 28$ & 0.095 & $-3 \cdot 74,46 \cdot 30$ & 18.01 & $0 \cdot 140$ & $-5 \cdot 96,41 \cdot 98$ & $-25 \cdot 29$ & 0.036 & $-48 \cdot 88,-1 \cdot 70$ & $-29 \cdot 31$ & 0.021 & $-54 \cdot 15,-4 \cdot 48$ \\
\hline 2 or 3 times per week & $45 \cdot 75$ & 0.001 & $20 \cdot 20,71 \cdot 29$ & $39 \cdot 53$ & 0.001 & $15 \cdot 39,63 \cdot 67$ & $-17 \cdot 96$ & $0 \cdot 138$ & $-41 \cdot 74,5 \cdot 82$ & $-23 \cdot 28$ & 0.065 & $-48 \cdot 03,1 \cdot 46$ \\
\hline Daily & $87 \cdot 58$ & 0.007 & $24 \cdot 14,151 \cdot 03$ & $80 \cdot 51$ & 0.025 & $10 \cdot 06,150 \cdot 95$ & $10 \cdot 89$ & 0.481 & $-19 \cdot 58,41 \cdot 35$ & 0.98 & 0.955 & $-33 \cdot 03,35 \cdot 00$ \\
\hline \multicolumn{13}{|c|}{ Frequency of fruit availability at home } \\
\hline Never & Reference & - & - & Reference & - & - & Reference & - & - & Reference & - & - \\
\hline Weekly & $51 \cdot 68$ & $<0.001$ & $26 \cdot 02,77 \cdot 34$ & $48 \cdot 85$ & $<0.001$ & $28 \cdot 26,69 \cdot 44$ & $13 \cdot 02$ & 0.612 & $-37 \cdot 52,63 \cdot 56$ & $9 \cdot 82$ & $0 \cdot 693$ & $-39 \cdot 21,58 \cdot 84$ \\
\hline Most of the time & $115 \cdot 31$ & $<0.001$ & $97 \cdot 24,133 \cdot 38$ & $100 \cdot 42$ & $<0 \cdot 001$ & $86 \cdot 16,114 \cdot 68$ & $21 \cdot 97$ & $0 \cdot 358$ & $-25 \cdot 15,69 \cdot 08$ & $9 \cdot 21$ & $0 \cdot 691$ & $-36 \cdot 54,54 \cdot 96$ \\
\hline \multicolumn{13}{|l|}{ Purchase organic food } \\
\hline No & eference & - & - & Reference & - & - & Reference & - & - & Reference & - & - \\
\hline Yes & $31 \cdot 74$ & $<0.001$ & $17 \cdot 11,46 \cdot 38$ & $24 \cdot 77$ & 0.001 & $9 \cdot 87,39 \cdot 66$ & $19 \cdot 52$ & 0.001 & $8 \cdot 01,31 \cdot 03$ & $14 \cdot 56$ & 0.019 & $2 \cdot 44,26.68$ \\
\hline \multicolumn{13}{|l|}{ Frequency of eating takeaway } \\
\hline Rarely or never & Reference & - & - & Reference & - & - & Reference & - & - & Reference & - & - \\
\hline Once or twice per month & $-18 \cdot 74$ & $0 \cdot 012$ & $-33 \cdot 24,-4 \cdot 23$ & $-14 \cdot 69 \ddagger$ & 0.052 & $-29 \cdot 48,0 \cdot 10$ & $-7 \cdot 10$ & 0.329 & $-21 \cdot 41,7 \cdot 22$ & $-4 \cdot 33$ & 0.549 & $-18 \cdot 54,9 \cdot 89$ \\
\hline More than once per week & $-39 \cdot 16$ & $<0.001$ & $-57 \cdot 55,-20 \cdot 77$ & $-32 \cdot 20$ & 0.001 & $-51 \cdot 64,-12 \cdot 76$ & $-25 \cdot 14$ & 0.007 & $-43 \cdot 38,-6 \cdot 90$ & $-20 \cdot 36$ & 0.028 & $-38 \cdot 46,-2 \cdot 27$ \\
\hline \multicolumn{13}{|l|}{ Frequency of eating out } \\
\hline Rarely or never & Reference & - & - & Reference & - & - & Reference & - & - & Reference & - & - \\
\hline Once or twice per month & $13 \cdot 67$ & $0 \cdot 130$ & $-4 \cdot 08,31 \cdot 43$ & $9 \cdot 96$ & $0 \cdot 271$ & $-7 \cdot 87,27 \cdot 79$ & $5 \cdot 57$ & 0.461 & $-9 \cdot 31,20 \cdot 44$ & $2 \cdot 56$ & $0 \cdot 732$ & $-12 \cdot 17,17 \cdot 29$ \\
\hline More than once per week & $8 \cdot 02$ & $0 \cdot 489$ & $-14 \cdot 81,30 \cdot 85$ & $4 \cdot 80$ & 0.665 & $-17 \cdot 07,26 \cdot 67$ & $1 \cdot 49$ & 0.875 & $-17 \cdot 20,20 \cdot 18$ & $-1 \cdot 33$ & $0 \cdot 889$ & $-20 \cdot 10,17 \cdot 45$ \\
\hline
\end{tabular}

FV, fruit and vegetable(s); MFP, main food provider; NSSEC5, National Statistic Socio-economic Classification 5 .

*Model adjusted for age and sex.

tModel adjusted for age, sex and NSSEC5.

$\ddagger$ †ariable category non-significant after adjusting for NSSEC5. 
Table 5 Eigenvalues, percentage of variance and the component loadings for the three patterns retained

\begin{tabular}{|c|c|c|c|}
\hline & Pattern 1 & Pattern 2 & Pattern 3 \\
\hline Eigenvalue & $2 \cdot 243$ & $1 \cdot 683$ & $1 \cdot 180$ \\
\hline$\%$ of variance ${ }^{\star}$ & $20 \cdot 4$ & $15 \cdot 3$ & $10 \cdot 7$ \\
\hline \multicolumn{4}{|l|}{ Component loadings for each pattern } \\
\hline Household size & $0.5817 t$ & $-0.3394 \dagger$ & 0.0603 \\
\hline Number of adults per household & $0.4278 t$ & -0.0106 & 0.2346 \\
\hline Number of children per household & $0.4280 t$ & $-0.4233+$ & $-0 \cdot 1021$ \\
\hline NSSEC5 & 0.2245 & $0.4715 t$ & -0.0615 \\
\hline Ethnicity & 0.0497 & $-0 \cdot 1645$ & $-0.3077 \dagger$ \\
\hline MFP age & $0 \cdot 2997$ & 0.2336 & 0.2232 \\
\hline Frequency of FV purchase & 0.2080 & $0 \cdot 2456$ & -0.2697 \\
\hline Frequency of fruit availability & $0 \cdot 2224$ & $0.3007 \dagger$ & -0.0941 \\
\hline Ever bought organic food & $0 \cdot 2384$ & $0.3547 \dagger$ & -0.0830 \\
\hline Frequency of eating out & 0.0047 & 0.2880 & $0.5496 t$ \\
\hline Frequency of eating takeaway & -0.0563 & -0.2049 & $0 \cdot 6261 \dagger$ \\
\hline
\end{tabular}

NSSEC5, National Statistic Socio-economic Classification 5; MFP, main food provider; FV, fruit and vegetable(s); SFP, sociodemographic and food purchase; PCA, principal component analysis.

*Percentage of variance explained by SFP variables in the PCA pattern scores.

tComponent loading of variable $\geq \pm 0 \cdot 30$ considered to be highly correlated with pattern.

Table 6 Regression of pattern scores for patterns 1,2 and 3 on fruit consumption $(\mathrm{g} / \mathrm{d})$ and vegetable consumption ( $\mathrm{g} / \mathrm{d})$ : children $(n$ 642) aged 1.5-10 years, UK National Diet and Nutrition Survey Rolling Programme (2008-2010)

\begin{tabular}{|c|c|c|c|c|c|c|}
\hline & \multicolumn{3}{|c|}{ Fruit } & \multicolumn{3}{|c|}{ Vegetables } \\
\hline & $\beta^{*}$ & $P$ value & $95 \% \mathrm{Cl}$ & $\beta$ & $P$ value & $95 \% \mathrm{Cl}$ \\
\hline Pattern 1 & $9 \cdot 51$ & $<0.001$ & $4 \cdot 82,14 \cdot 20$ & 0.82 & 0.709 & $-3 \cdot 50,5 \cdot 14$ \\
\hline Pattern 2 & $16 \cdot 81$ & $<0.001$ & $12 \cdot 15,21 \cdot 48$ & 8.08 & $<0.001$ & $3 \cdot 71,12 \cdot 45$ \\
\hline Pattern 3 & $-8 \cdot 63$ & 0.012 & $-15 \cdot 38,-1 \cdot 89$ & $-7 \cdot 23$ & 0.023 & $-13.47,-0.99$ \\
\hline
\end{tabular}

${ }^{*} \beta$, beta coefficient representing an increase of consumption $(\mathrm{g} / \mathrm{d})$ per unit increase of pattern score.

children, where children of mothers aged less than 25 years were less likely to eat fruit and while those of mothers aged 25-29 years were less likely to eat vegetables ${ }^{(38)}$. Older MFP may have greater knowledge and experience in providing food for children than those younger and less experienced, thus making healthier choices for children compared with their younger counterparts. FV consumption in children was also predicted by organic food purchases. Organic foods are sometimes perceived to be superior and healthier than non-organic, even though they are also more expensive ${ }^{(39)}$. Organic buyers may be more conscious of the health of their children than those who never buy organic foods ${ }^{(40)}$.

We have demonstrated a novel approach to studying the combined effects of correlated SFP characteristics with FV consumption, using an exploratory data-driven technique, through which we identified three distinct patterns in NDNS children. The first pattern was characterised mainly by household structure variables. In the independent analysis, very few associations were seen between household structure characteristics and FV consumption; however as a pattern, household size was associated with fruit consumption, with children from larger households having higher fruit consumption. The results suggested that a higher ratio of parents to children in the household may be more important to predict children's fruit consumption than just the size of household alone. The second pattern was also of considerable interest, as it corresponded well with a combination of household factors and food practice variables. In contrast to the first pattern regarding household size, the pattern described a fewer number of children from smaller households. It also described higher-SES households with greater fruit availability and organic food purchases. These characteristics reflected households with greater affordability of fruit and vegetables for their children's diet, and were related to higher FV consumption. The third pattern was driven by the practice of a high frequency of eating out and takeaway food. Although the independent association of eating out was not significant, the pattern suggested that the overall behaviour of frequent eating out and takeaway was unfavourable for both fruit consumption and vegetable consumption. This finding resonates with previous literature on fast food and takeaway and diet quality ${ }^{(41,42)}$.

The strength of the present study lies in the high quality of the data, collected from a nationally representative sample of UK children aged $1 \cdot 5-10$ years, using $4 \mathrm{~d}$ diet diaries and comprehensive interviews delivered by trained interviewers. The measurement of FV consumption after disaggregation provided more complete dietary data than other studies ${ }^{(24)}$. The use of PCA on SFP data advances knowledge of determinants of FV consumption within the 
home setting, demonstrating combined effects of SFP characteristics in relation to $\mathrm{FV}$ consumption. A further advantage of PCA was to minimise the problem of reduced statistical power of highly correlated factors analysed together; we have demonstrated the differences in analysing the SFP characteristics using independent regression with adjustment of household SES $v$. patterns of these characteristics on FV consumption in children.

There are, however, limitations associated with the study. PCA is a data-driven technique and the patterns produced are dependent on the data collected and sample specific. If the same technique is applied to another sample, the patterns produced may not be directly comparable to our results. PCA is known to be inflexible in dealing with measurement error-prone data, as well as non-continuous or ordinal data. As a result, only certain variables were analysed in the current study. Another limitation is the use of NSSEC5 as proxy of SES. Although NSSEC5 is considered a superior measure of household SES compared with measures used in previous national surveys, it does not reflect other important aspects of SES including household income and parental education. These have been shown to impact eating behaviour and dietary intake and therefore may have contributed to residual confounding in the results. Furthermore, because NDNS is nationally representative of the UK population, the majority of children in the current study were white. In addition, the children sample was from relatively high socio-economic groups. These factors may have limited the findings and the patterns derived from PCA, as previous studies have suggested that low consumption due to low availability and accessibility of fruit and vegetables tends to occur in those from lower socio-economic backgrounds and possibly in ethnic minority groups.

The study provides new insights on relationships between patterns of SFP characteristics in relation to FV consumption in a nationally representative sample of UK children, and has shown combined effects of these characteristics on FV consumption. While the method used was exploratory and the results may not apply to other populations, the findings add value to current perspectives on determinants of children's diet quality at the household level. Furthermore, the results have implications for public health practice and policy. Households who eat out and eat takeaways frequently are at higher risks of low FV consumption. The government should encourage food industry and caterers to increase FV content in ready meals and takeaways, and to provide more meal options with fruit and vegetables in restaurants and other eating establishments. These are likely to benefit families who often eat in such settings. Smaller families with high parent-to-children ratios from more superior socioeconomic backgrounds are in a better position to provide fruit and vegetables for their children. Although family size or composition cannot be intervened or changed, it is important for public health nutrition policy makers to target families of lower SES to reduce such diet inequality. Possible ways for intervention could be to subsidise the costs of fruit and vegetables for families in low socio-economic positions, as well as to concentrate efforts on families with low income-to-family size ratios.

\section{Acknowledgements}

Sources of funding: The National Diet and Nutrition Survey is funded by the Department of Health and the Food Standards Agency. The present study was supported by the UK Medical Research Council (MRC project no. MC_US_A090_0039). T.N.M. was supported by a Cambridge International Student Scholarship from the Cambridge Overseas Trust. Conflicts of interest: The authors declare no conflicts of interest. Authors' contributions: T.N.M. initiated the study, developed the methodology, analysed and interpreted the data, and wrote the manuscript. C.J.P., D.C. and T.N.M. prepared the dietary data. B.B. provided the CAPI data. A.M.S. oversaw the NDNS dietary data collection, coding and analysis, and supervised and edited the manuscript. All authors read and approved the final manuscript.

\section{References}

1. Burke SJ, McCarthy SN, O'Neill JL et al. (2007) An examination of the influence of eating location on the diets of Irish children. Public Health Nutr 10, 599-607.

2. Ding D, Sallis JF, Norman GJ et al. (2012) Community food environment, home food environment, and fruit and vegetable intake of children and adolescents. J Nutr Educ Behav 44, 634-638.

3. Arcan C, Neumark-Sztainer D, Hannan P et al. (2007) Parental eating behaviours, home food environment and adolescent intakes of fruits, vegetables and dairy foods: longitudinal findings from Project EAT. Public Health Nutr 10, 1257-1265.

4. Riediger ND, Shooshtari S \& Moghadasian MH (2007) The influence of sociodemographic factors on patterns of fruit and vegetable consumption in Canadian adolescents. J Am Diet Assoc 107, 1511-1518.

5. Wyse R, Campbell E, Nathan N et al. (2011) Associations between characteristics of the home food environment and fruit and vegetable intake in preschool children: a cross-sectional study. BMC Public Health 11, 938.

6. Vereecken C, Haerens L, De Bourdeaudhuij I et al. (2010) The relationship between children's home food environment and dietary patterns in childhood and adolescence. Public Health Nutr 13, 1729-1735.

7. McGowan L, Croker H, Wardle J et al. (2012) Environmental and individual determinants of core and non-core food and drink intake in preschool-aged children in the United Kingdom. Eur J Clin Nutr 66, 322-328.

8. Johnson L, van Jaarsveld CH \& Wardle J (2011) Individual and family environment correlates differ for consumption of core and non-core foods in children. Br J Nutr 105, 950-959.

9. Vereecken C \& Maes L (2010) Young children's dietary habits and associations with the mothers' nutritional knowledge and attitudes. Appetite 54, 44-51.

10. Ryden PJ \& Hagfors L (2011) Diet cost, diet quality and socio-economic position: how are they related and what contributes to differences in diet costs? Public Health Nutr 14, 1680-1692. 
11. Aggarwal A, Monsivais P, Cook AJ et al. (2011) Does diet cost mediate the relation between socioeconomic position and diet quality? Eur J Clin Nutr 65, 1059-1066.

12. Mullie P, Clarys P, Hulens M et al. (2010) Dietary patterns and socioeconomic position. Eur J Clin Nutr 64, 231-238.

13. McLeod ER, Campbell KJ \& Hesketh KD (2011) Nutrition knowledge: a mediator between socioeconomic position and diet quality in Australian first-time mothers. J Am Diet Assoc 111, 696-704.

14. Bihan H, Castetbon K, Mejean C et al. (2010) Sociodemographic factors and attitudes toward food affordability and health are associated with fruit and vegetable consumption in a low-income French population. J Nutr 140, 823-830.

15. Cutler GJ, Flood A, Hannan P et al. (2011) Multiple sociodemographic and socioenvironmental characteristics are correlated with major patterns of dietary intake in adolescents. I Am Diet Assoc 111, 230-240.

16. Borodulin K, Zimmer C, Sippola R et al. (2012) Health behaviours as mediating pathways between socioeconomic position and body mass index. Int $J$ Behav Med 19, 14-22.

17. Lv J, Liu Q, Ren Y et al. (2011) Socio-demographic association of multiple modifiable lifestyle risk factors and their clustering in a representative urban population of adults: a cross-sectional study in Hangzhou, China. Int $J$ Behav Nutr Phys Act 8, 40.

18. Poortinga W (2007) The prevalence and clustering of four major lifestyle risk factors in an English adult population. Prev Med 44, 124-128.

19. Department of Health \& Food Standards Agency (2011) National Diet and Nutrition Survey: Headline results from Years 1 and 2 (combined) of the Rolling Programme (2008/9-2009/10). http://www.dh.gov.uk/prod_consum_dh/ groups/dh_digitalassets/documents/digitalasset/dh_128550.pdf (accessed October 2011).

20. Food Standards Agency \& Department of Health (2010) National Diet and Nutrition Survey: Headline results from Year 1 of the Rolling Programme. Appendix B Weighting the NDNS core sample. http://tna.europarchive. org/20110116113217/http://www.food.gov.uk/multimedia/ pdfs/publication/ndns0809appendixb.pdf (accessed May 2011).

21. Food Standards Agency \& Department of Health (2010) National Diet and Nutrition Survey: Headline results from Year 1 of the Rolling Programme (2008/2009). http://tna. europarchive.org/20110116113217/http://www.food.gov.uk/ multimedia/pdfs/publication/ndnsreport0809.pdf (accessed April 2010)

22. Food Standards Agency \& Department of Health (2010) National Diet and Nutrition Survey: Headline results from Year 1 of the Rolling Programme. Appendix A Dietary data collection and editing. http://www.food.gov. uk/multimedia/pdfs/publication/ndns0809appendixa.pdf (accessed April 2010).

23. Food Standards Agency (2002) Food Portion Sizes, 3rd edn. London: The Stationery Office.

24. Fitt E, Mak TN, Stephen AM et al. (2010) Disaggregating composite food codes in the UK National Diet and Nutrition Survey food composition databank. Eur J Clin Nutr 64, Suppl. 3, S32-S36.

25. Food Standards Agency (2002) McCance and Widdowson's The Composition of Foods, 6th ed. Cambridge: Royal Society of Chemistry.

26. Department of Health \& Food Standards Agency (2011) National Diet and Nutrition Survey: Headline results from Years 1 and 2 (combined) of the Rolling Programme 2008/ 9-2009/10. Appendix D: Interviewer (stage 1) overview of elements and documents. http://www.dh.gov.uk/prod_ consum_dh/groups/dh_digitalassets/documents/digitalasset/ dh_128543.pdf (accessed July 2011).

27. Office for National Statistics (2010) The National Statistics Socio-economic Classification (NS-SEC rebased on the SOC2010). http://www.ons.gov.uk/ons/guide-method/ classifications/current-standard-classifications/soc2010/soc 2010-volume-3-ns-sec-rebased-on-soc2010-user-manual/ index.html (accessed February 2012).

28. Rose D, Pevalin DJ \& O'Reilly K (2005) The National Statistics Socio-economic Classification: Origins, Development and Use. London: Office for National Statistics.

29. Vyas S \& Kumaranayake L (2006) Constructing socioeconomic status indices: how to use principal components analysis. Health Policy Plan 21, 459-468.

30. Kolenikov S \& Angeles G (2009) Socioeconomic status measurement with discrete proxy variables: is principal component analysis a reliable answer? Rev Income Wealth 55, 128-165.

31. Cullen KW, Baranowski T, Owens E et al. (2003) Availability, accessibility, and preferences for fruit, 100\% fruit juice, and vegetables influence children's dietary behavior. Health Educ Behav 30, 615-626.

32. Campbell KJ, Crawford DA, Salmon J et al. (2007) Associations between the home food environment and obesity-promoting eating behaviors in adolescence. Obesity (Silver Spring) 15, 719-730.

33. Pearson N, Atkin AJ, Biddle SJH et al. (2010) Parenting styles, family structure and adolescent dietary behaviour. Public Health Nutr 13, 1245-1253.

34. Rasmussen M, Krolner R, Klepp K-I et al. (2006) Determinants of fruit and vegetable consumption among children and adolescents: a review of the literature. Part I: quantitative studies. Int J Behav Nutr Phys Act 3, 22.

35. Mak T, Prynne C, Cole D et al. (2012) Assessing eating context and fruit and vegetable consumption in children: new methods using food diaries in the UK National Diet and Nutrition Survey Rolling Programme. Int J Behav Nutr Phys Act 9, 126.

36. Reinaerts E, de Nooijer J, Candel M et al. (2007) Explaining school children's fruit and vegetable consumption: the contributions of availability, accessibility, exposure, parental consumption and habit in addition to psychosocial factors. Appetite 48, 248-258.

37. Brazionis L, Golley RK, Mittinty MN et al. (2012) Characterization of transition diets spanning infancy and toddlerhood: a novel, multiple-time-point application of principal components analysis. Am J Clin Nutr 95, 1200-1208.

38. Rogers I \& Emmett P (2003) The effect of maternal smoking status, educational level and age on food and nutrient intakes in preschool children: results from the Avon Longitudinal Study of Parents and Children. Eur J Clin Nutr 57, 854-864.

39. Makatouni A (2002) What motivates consumers to buy organic food in the UK?: results from a qualitative study. $\mathrm{Br}$ Food J 104, 345-352.

40. Davies A, Titterington A \& Cochrane C (1995) Who buys organic food?: a profile of the purchasers of organic food in Northern Ireland. Br Food J 97, 17-23.

41. Timperio A, Ball K, Roberts R et al. (2008) Children's fruit and vegetable intake: associations with the neighbourhood food environment. Prev Med 46, 331-335.

42. Bowman SA, Gortmaker SL, Ebbeling CB et al. (2004) Effects of fast-food consumption on energy intake and diet quality among children in a National Household Survey. Pediatrics 113, 112-118. 